\title{
Analytical Solutions for Testing Ray-Effect Errors in Numerical Solutions of the Transport Equation
}

\author{
B. Chang
}

U.S. Department of Energy

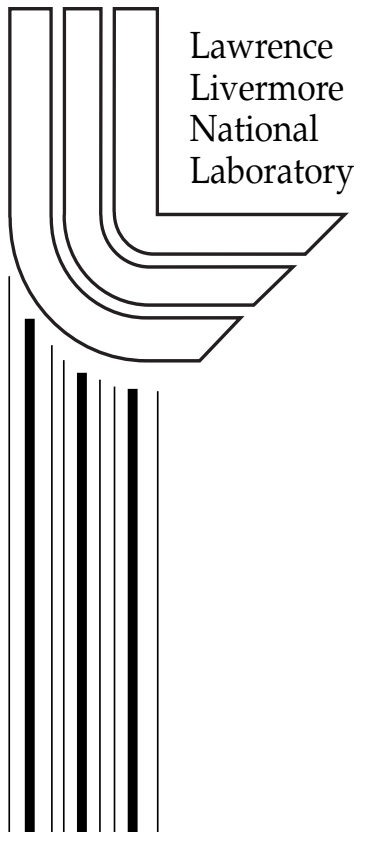

May 12, 2003 
This document was prepared as an account of work sponsored by an agency of the United States Government. Neither the United States Government nor the University of California nor any of their employees, makes any warranty, express or implied, or assumes any legal liability or responsibility for the accuracy, completeness, or usefulness of any information, apparatus, product, or process disclosed, or represents that its use would not infringe privately owned rights. Reference herein to any specific commercial product, process, or service by trade name, trademark, manufacturer, or otherwise, does not necessarily constitute or imply its endorsement, recommendation, or favoring by the United States Government or the University of California. The views and opinions of authors expressed herein do not necessarily state or reflect those of the United States Government or the University of California, and shall not be used for advertising or product endorsement purposes.

This work was performed under the auspices of the U.S. Department of Energy by University of California, Lawrence Livermore National Laboratory under Contract W-7405-Eng-48. 


\title{
ANALYTICAL SOLUTIONS FOR TESTING RAY-EFFECT ERRORS IN NUMERICAL SOLUTIONS OF THE TRANSPORT EQUATION
}

\author{
BRITTON CHANG *
}

1. Introduction. This paper contains three analytical solutions of transport problems which can be used to test ray-effect errors in the numerical solutions of the Boltzmann Transport Equation (BTE). We derived the first two solutions and the third was shown to us by M. Prasad [2]. Since this paper is intended to be an internal LLNL report, no attempt was made to find the original derivations of the solutions in the literature in order to cite the authors for their work.

Before we embarked on this project, the only analytical solution known to us which could serve as a diagnostic test for ray-effects is the solution of an isotropic point source in a homogeneous non-scattering medium. However this solution is not an accurate test, because a point source is difficult to represent numerically. Thus it is useful for testing purposes to have an analytical solution of a localized volume source. The sources in the three problems below are isotropic spheres centered at the origin. Since ray-effect oscillations are the most pronounced for the transport in a vacuum (because the oscillations are not damped out by absorption), the absorption is set to zero in the regions exterior of the sources in the three problems. Since these problems are spherically symmetric, they are solved in the 1-D spherical coordinate system.

2. Problem A. Problem A is an isotropic source in a vacuum. The source is a sphere of radius $a$ and the vacuum is a sphere of radius $b$ with $b>a$. The absorption is everywhere zero. The transport equation for this problem is

$$
\text { Problem A : } \quad \Omega \cdot \nabla \psi=\frac{\mathrm{q}}{4 \pi}, \quad \text { where } \quad \mathrm{q}= \begin{cases}1, & r \leq a \\ 0, & r>a,\end{cases}
$$

with the boundary condition

$$
\psi(r=b, \mu \leq 0)=0 .
$$

The streaming operator in the 1-D spherical coordinate system is [1]

$$
\Omega \cdot \nabla \equiv \mu \frac{\partial}{\partial r}+\frac{1-\mu^{2}}{r} \frac{\partial}{\partial \mu}
$$

where $\mu \equiv \Omega \cdot \mathbf{r} / r$ is the cosine of the angle between the neutron's direction $\Omega$ and the position vector $\mathbf{r}$, and $r=|\mathbf{r}|$ is the distance between the position $\mathbf{r}$ and the origin.

Equation (2.1) is solved by the method of characteristics, and (2.1) in characteristic form is

$$
\frac{d \psi}{d s}=\frac{q}{4 \pi},
$$

${ }^{*}$ Center for Applied Scientific Computing, Lawrence Livermore National Laboratory, P.O. Box 808 L-561, Livermore, CA 94551. email: bchang@llnl.gov. This work was performed under the auspices of the U.S. Department of Energy by Lawrence Livermore National Laboratory under contract no. W-7405-Eng-48. 


$$
\begin{aligned}
& \frac{d r}{d s}=\mu, \\
& \frac{d \mu}{d s}=\frac{1-\mu^{2}}{r} .
\end{aligned}
$$

The characteristic equations (2.3) are solved by deriving a functional relationship between $r$ and $\mu$. The differential equation for this functional relationship is determined by dividing the second equation of (2.3) by the third equation of (2.3)

$$
\frac{d r}{d \mu}=\frac{r \mu}{1-\mu^{2}} .
$$

Equation (2.4) can be integrated easily to yield

$$
r \sqrt{1-\mu^{2}}=r_{2} \sqrt{1-\mu_{2}^{2}},
$$

where $r_{2}$ and $\mu_{2}$ are the integration constants that characterizes a path in $(r, \mu)$ space. Let these integration constants $r_{2} \equiv r\left(s_{2}\right)$ and $\mu_{2} \equiv \mu\left(s_{2}\right)$ be the final conditions of $r$ and $\mu$ respectively.

Solving (2.5) for $\mu$ and substituting the result into the second equation of (2.3) gives a differential equation for $r$ with a right hand side (rhs) that depends on $r$ only. This equation can be integrated analytically from $s_{0}$ to $s_{2}$. Similarly, a differential equation for $\mu$ with a rhs that depends on $\mu$ only can be derived by solving (2.5) for $r$ and substituting the result into the third equation of (2.3). This differential equation can also be integrated analytically from $s_{0}$ to $s_{2}$. The integrals are

$$
\begin{aligned}
& r_{0}^{2}=\left(1-\mu_{2}^{2}\right) r_{2}^{2}+\left(\mu_{2} r_{2}-\left(s_{2}-s_{0}\right)\right)^{2}, \\
& \mu_{0}=\frac{\mu_{2} r_{2}-\left(s_{2}-s_{0}\right)}{r_{0}},
\end{aligned}
$$

where $r_{0} \equiv r\left(s_{0}\right), \mu_{0} \equiv \mu\left(s_{0}\right)$ is the point on the inflow boundary $\left(r_{0}=b, \mu_{0}<0\right)$ that the characteristic intersects in the 'backward direction' from $\left(r_{2}, \mu_{2}\right)$, and where $s_{2}-s_{0}$ is the path length between $\left(r_{0}, \mu_{0}\right)$ and $\left(r_{2}, \mu_{2}\right)$. The formal solution of the first equation of $(2.3)$ is

$$
\psi\left(r_{2}, \mu_{2}\right)-\psi\left(r_{0}, \mu_{0}\right)=\int_{s_{0}}^{s_{2}} \frac{q(r(s))}{4 \pi} d s .
$$

An understanding of the types of path that $(2.6)$ generates in $(r, \mu)$ space helps to solve the path integral in (2.7). Drawn in Fig. (2.1) are three of types of paths. The path type depends on the initial condition $\left(r_{0}, \mu_{0}\right)$. Path $C$ is the ' $U$ ' shaped arc $C_{0} C_{1} C_{2}$ that starts from the boundary $r=b$ at $C_{0}$, reaches its lowest point at $C_{1}$, turns upward, and then exits the boundary at $C_{2}$ without passing through the source region $r \leq a$. On the other hand, path $A$ starts from $A_{0}$, passes through the source at the entry and the exit points $A_{1}$ and $A_{3}$ respectively, and then exits the domain at $A_{4}$. Path B grazes the source at $B_{1}$. Since the paths above path B do not intersect the source while the paths below path B intersect the source, then path $\mathrm{B}$ can be considered to be the separatrix that separates the paths that intersect the source from those that do not. Therefore $\psi$ in the region which is above the separatrix $\mathrm{B}$ is identically zero, because the boundary condition (2.2) of $\psi$ at the starting points 


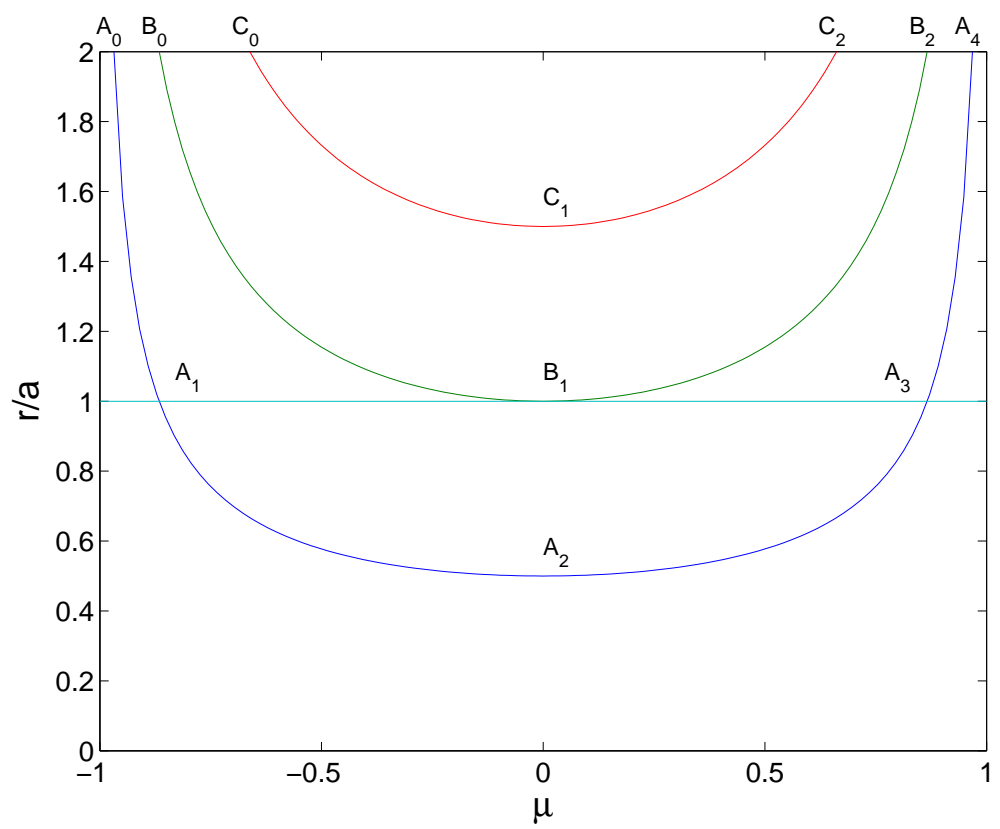

FIG. 2.1. Characteristic paths in $(r, \mu)$ space for the domain $0 \leq r \leq 2 a$

of the paths in this region is zero, and the source integrals in (2.7) for these paths are also zero.

Consider the region $r \leq a$. Let $\left(r_{2}, \mu_{2}\right)$ be such a point in this region, e.g. the point $A_{2}$ in Fig. (2.1). Tracing backwards from $A_{2}$ with (2.6), let $\left(r_{0}, \mu_{0}\right)$ be the point on the boundary which the characteristic intersects, i.e. $A_{0}$. Furthermore let $s_{1}$ be the path parameter for the point $A_{1}, r\left(s_{1}\right)=a, \mu_{1} \equiv \mu\left(s_{1}\right)$. Then (2.7) for the path $A_{0} A_{1} A_{2}$ is

$$
\psi\left(r_{2}, \mu_{2}\right)=\int_{s_{0}}^{s_{2}} \frac{q(r(s))}{4 \pi} d s=\int_{s_{1}}^{s_{2}} \frac{q(r(s))}{4 \pi} d s=\frac{1}{4 \pi}\left(s_{2}-s_{1}\right),
$$

since $\psi\left(r_{0}=b, \mu_{0} \leq 0\right)=0$ by $(2.2), q=0$ in the segment $A_{0} A_{1}$, and $q=1$ in the segment $A_{1} A_{2}$.

The path length $s_{2}-s_{1}$ between the points $A_{1}$ and $A_{2}$ can be determined as follows. Since (2.6) is a general equation for any pair of points on the characteristic $A_{0} A_{1} A_{2}$, then it holds for $A_{1}$ and $A_{2}$. Replacing $r_{0}, \mu_{0}$ and $s_{0}$ in (2.6) by $r_{1}, \mu_{1}$, and $s_{1}$ respectively gives a relationship between the points $A_{1}$ and $A_{2}$ in terms of $s_{2}-s_{1}$. The path length $s_{2}-s_{1}$ is found by inverting the first of these equations with the left hand side set equal to $a$

$$
s_{2}-s_{1}=\mu_{2} r_{2}+\sqrt{a^{2}-\left(1-\mu_{2}^{2}\right) r_{2}^{2}} .
$$

Thus by (2.8) and (2.9), $\psi$ in region $r \leq a$ is explicitly

$$
\psi(r \leq a, \mu)=\frac{\mu r+\sqrt{a^{2}-\left(1-\mu^{2}\right) r^{2}}}{4 \pi} .
$$

Turning to the region outside of the source $r>a$. The separatrix B divides this region into three subregions according to a criterion that depends on $\mu$. The separatrix 
is also needed to derive $\psi$ in these subregions. The equation for the separatrix is determined from (2.5) the functional relationship between $r$ and $\mu$. Since any pair of points on the arc $B_{0} B_{1} B_{2}$ is related by (2.5), then let $B_{1}$ be the reference point $\left(r_{2}, \mu_{2}\right)$ in (2.5). Substituting $r_{2}=a$ and $\mu_{2}=0$, the $(r, \mu)$ coordinates of $B_{1}$ (see Fig. (2.1) for reference), into (2.5) yields $r \sqrt{1-\mu^{2}}=a$ for the separatrix. A more useful form for the equation of the separatrix is

$$
\mu= \pm \sqrt{1-\frac{a^{2}}{r^{2}}}
$$

For a point $r$ which is larger than $a,(2.11)$ divides this region into three subregions; the subregion $-1 \leq \mu \leq-\left(1-a^{2} / r^{2}\right)^{1 / 2}$, the subregion $-\left(1-a^{2} / r^{2}\right)^{1 / 2}<\mu<$ $\left(1-a^{2} / r^{2}\right)^{1 / 2}$, and the subregion $\left(1-a^{2} / r^{2}\right)^{1 / 2} \leq \mu \leq 1$. The angular flux $\psi$ is independent of the source in the first two subregions, because the paths in these subregions do not intersect the source (see Fig. 2.1 for reference). Since the boundary condition (2.2) contributes nothing to the angular flux in (2.7), the angular flux $\psi$ is zero in these two subregions. Since a path from the inflow boundary to a point in the subregion $\mu>\left(1-a^{2} / r^{2}\right)^{1 / 2}$ crosses the source region, then the source integral of (2.7) and hence $\psi$ are non-zero only if $\mu>\left(1-a^{2} / r^{2}\right)^{1 / 2}$ for $r>a$.

Let us calculate $\psi$ for such a point, e.g. $A_{4}$. Let $s_{0}, s_{1}, s_{3}$, and $s_{4}$ be the path parameters of $A_{0}, A_{1}, A_{3}$ and $A_{4}$ respectively. It is clear from (2.7) that

$$
\psi\left(r_{4}, \mu_{4}\right)=\int_{s_{0}}^{s_{4}} \frac{q(r(s))}{4 \pi} d s=\int_{s_{1}}^{s_{3}} \frac{q(r(s))}{4 \pi} d s,
$$

because the source is non-zero only on the path segment $A_{1} A_{2} A_{3}$. However the last integral in (2.12) was evaluated in (2.10) and is equal to the rhs of $\psi\left(r_{3}, \mu_{3}\right)$ in $(2.10)$. Thus

$$
\psi\left(r_{4}, \mu_{4}\right)=\psi\left(r_{3}, \mu_{3}\right)=\frac{\mu_{3} a+\left|\mu_{3}\right| a}{4 \pi} .
$$

The equivalence of $\psi\left(r_{4}, \mu_{4}\right)$ and $\psi\left(r_{3}, \mu_{3}\right)$ in $(2.13)$ is the mathematical statement of the conservative nature of the solution of a hyperbolic equation, i.e. that $\psi$ in a sourceless void is constant on a characteristic.

In order for (2.13) to be a useful solution for $\psi\left(r_{4}, \mu_{4}\right), \mu_{3}$ needs to be expressed in terms of $r_{4}$ and $\mu_{4}$. This expression is given by $(2.5), r_{3} \sqrt{1-\mu_{3}^{2}}=r_{4} \sqrt{1-\mu_{4}^{2}}$. The substitution $r_{3}=a$ into this equation yields $\mu_{3}=\left(1-\left(1-\mu_{4}^{2}\right) r_{4}^{2} / a^{2}\right)^{1 / 2}$. Using the identity $\mu+|\mu|=2 \mu H(\mu)$, where $H$ is the Heaviside function

$$
H(\xi)=\left\{\begin{array}{cc}
0, & \xi \leq 0 \\
1, & \xi>0
\end{array}\right.
$$

then $\psi$, in the region $r>a$ and $\mu>\left(1-a^{2} / r^{2}\right)^{1 / 2}$, is

$$
\psi(r>a, \mu)=\frac{2}{4 \pi} \sqrt{a^{2}-\left(1-\mu^{2}\right) r^{2}} H\left(\mu-\sqrt{1-\frac{a^{2}}{r^{2}}}\right) .
$$

Therefore an easy calculation for the scalar flux, $\phi(r) \equiv 2 \pi \int_{-1}^{1} \psi(r, \mu) d \mu$, gives

$$
\phi(r)=\frac{1}{2} a+\frac{a^{2}-r^{2}}{4 r} \log _{e} \frac{r+a}{|r-a|}, \quad \forall r .
$$


3. Problem B. Problem A is not very realistic because it is absorption-less. The very fact that it is absorption-less makes it a stringent test for ray effect errors in a numerical solution. A more realistic model is to put an absorber in the source region. In photon transport for example the following equation,

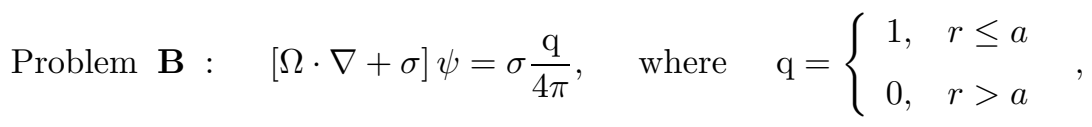

and the boundary condition (2.2) model a ball which emits photons isotropically into a vacuum. Let the absorption cross section be large in the source region and be zero outside the source, i.e.

$$
\sigma=\left\{\begin{array}{cc}
\frac{\sigma_{0}}{a}, & r \leq a \\
0, & r>a
\end{array}, \text { where } \sigma_{0}=1000\right.
$$
form is

Equation (3.1) is solved by the method of characteristic, and it in characteristic

$$
\frac{d \psi}{d s}+\sigma \psi=\sigma \frac{q}{4 \pi}
$$

The integral form of (3.3) is

$$
\psi\left(r_{2}, \mu_{2}\right)=e^{-\tau\left(s_{2}, s_{0}\right)} \psi\left(r_{0}, \mu_{0}\right)+\frac{1}{4 \pi} \int_{s_{0}}^{s_{2}} e^{-\tau\left(s_{2}, s_{0}\right)+\tau\left(s, s_{0}\right)} \sigma(r(s)) q(r(s), \mu(s)) d s
$$

where $\tau$ the optical depth is the path integral

$$
\tau\left(s_{2}, s_{0}\right) \equiv \int_{s_{0}}^{s_{2}} \sigma(r(s)) d s .
$$

Note that $\psi\left(r_{0}, \mu_{0}\right)=0$ because of the boundary condition (2.2).

Consider the source region $r \leq a$ first. Since $\sigma$ is constant inside the source region $r \leq a$ and is zero elsewhere, the path integral in (3.5) is essentially the same kind of path integral that is in $\S 2$. For example the optical depth on the arc $A_{0} A_{1} A_{2}$ in Fig. 2.1 is $\tau\left(s_{2}, s_{0}\right)=\tau\left(s_{2}, s_{1}\right)=\left(\sigma_{0} / a\right)\left(s_{2}-s_{1}\right)$, where the path length $s_{2}-s_{1}$ is given by (2.9). Thus the optical depth inside the source region $r \leq a$ is

$$
\tau\left(s_{2}, s_{1}\right)=\frac{\sigma_{0}}{a}\left(\mu_{2} r_{2}+\sqrt{a^{2}-\left(1-\mu_{2}^{2}\right) r_{2}^{2}}\right) .
$$

It is also easy to show that the path integral of (3.4) on the $\operatorname{arc} A_{0} A_{1} A_{2}$ gives $\psi=$ $\frac{1}{4 \pi}\left(1-e^{-\left(\sigma_{0} / a\right)\left(s_{2}-s_{1}\right)}\right)$. As a result of this expression and (3.6), the angular flux is

$$
\psi(r \leq a, \mu)=\frac{1}{4 \pi}\left(1-e^{-\frac{\sigma_{0}}{a}\left(\mu r+\sqrt{a^{2}-\left(1-\mu^{2}\right) r^{2}}\right)}\right) .
$$

In the region $r>a, \psi(r>a, \mu)$ is non-zero only if $\mu>\left(1-a^{2} / r^{2}\right)^{1 / 2}$, because the paths in this subregion emanate from the source. Since this region is a vacuum and is sourceless, then the fact that $\psi$ is constant on a characteristic can be used to determine $\psi$ in this region from its value on the surface $r=a$. For example on the 

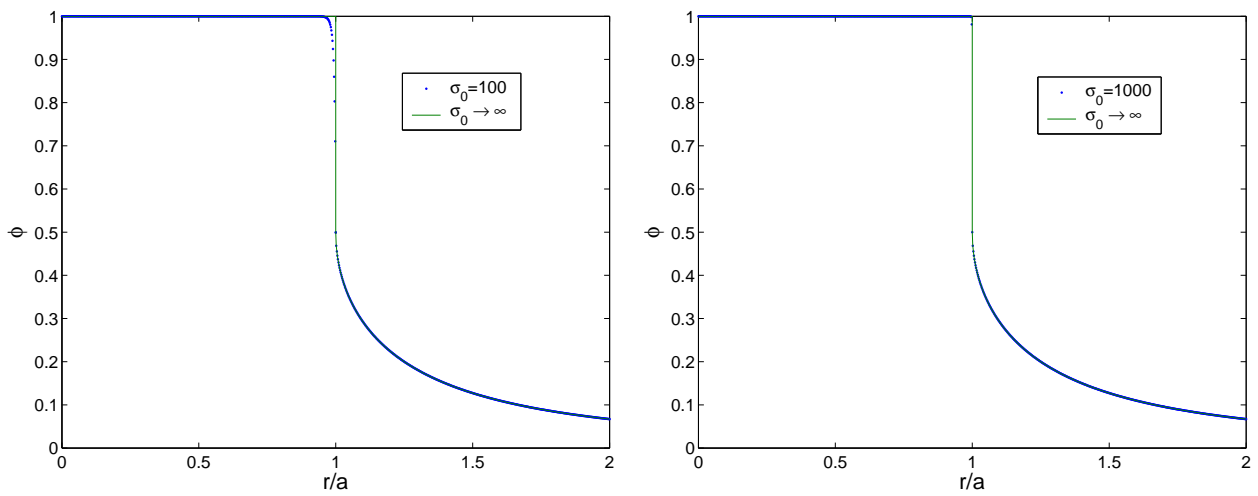

FIG. 3.1. A comparison of the $\sigma_{0} \rightarrow \infty$ limit with the two cases $\sigma_{0}=100$, and 1000 .

$\operatorname{arc} A_{3} A_{4}, \psi\left(r_{4}, \mu_{4}\right)=\psi\left(r_{3}, \mu_{3}\right)$, but $\psi\left(r_{3}, \mu_{3}\right)$ is given by (3.7). Hence it is easy to verify that

$$
\psi(r>a, \mu)=\frac{1}{4 \pi}\left(1-e^{-\frac{\sigma_{0}}{a}\left(2 \sqrt{a^{2}-\left(1-\mu^{2}\right) r^{2}}\right)}\right) H\left(\mu-\sqrt{1-\frac{a^{2}}{r^{2}}}\right) .
$$

I do not know any analytical expression for the $\mu$ integrals of (3.7) nor of (3.8). However, in the $\sigma_{0} \rightarrow \infty$ limit, (3.7) and (3.8) simplify to

$$
\psi_{\infty}(r, \mu)=\frac{1}{4 \pi}\left\{\begin{array}{cl}
1, & r<a \\
H\left(\mu-\sqrt{1-\frac{a^{2}}{r^{2}}}\right), & r \geq a .
\end{array}\right.
$$

The scalar flux $\phi(r) \equiv 2 \pi \int_{-1}^{1} \psi_{\infty}(r, \mu) d \mu$ in this limit is

$$
\phi(r)=\frac{1}{2}\left\{\begin{array}{cc}
2, & r<a \\
\left(1-\sqrt{1-\frac{a^{2}}{r^{2}}}\right), & r \geq a .
\end{array}\right.
$$

The angular flux of (3.7) and (3.8) is integrated with respect to $\mu$ by the mid-point quadrature for the cases $\sigma_{0}=100$ and $\sigma_{0}=1000$. These results are plotted against the $\sigma_{0} \rightarrow \infty$ limit (3.10) in Fig. (3.1). The disagreement between the $\sigma_{0}=100$ case and the $\sigma_{0}=\infty$ limit is localized to the region of few mean free paths below the surface of the sphere, and is of the order of a few percent. The relative error between the $\sigma_{0}=1000$ case and the $\sigma_{0}=\infty$ limit is everywhere less than .01 percent.

4. Problem C. When $\sigma$ is very large, then as argued in the last problem the photons do not travel very far from where they are emitted. The photons that are born deep in the interior of the source do not make it out of the source region. Thus the only photons that leave the source region and that determine $\psi$ in the region $r>a$ are those that are emitted within one mean free path of the surface of the source $r=a$. In the limit that $\sigma_{0}=\infty$ in (3.1), the source in Problem B can be replaced by a boundary condition at $r=a$, and the domain $0 \leq r \leq b$ can also be replaced by the domain $a \leq r \leq b$. Thus the transport equation for Problem $\mathbf{C}$ is

$$
\text { Problem } \mathbf{C}: \quad \Omega \cdot \nabla \psi=0, \quad \psi(\mathrm{r}=\mathrm{a}, \mu>0)=\psi_{\mathrm{a}}(\mu), \quad \psi(\mathrm{r}=\mathrm{b}, \mu<0)=0 .
$$


Since $\psi$ is constant on a characteristic, then the solution of (4.1) can be written down by inspection

$$
\psi(r, \mu)=\psi_{a}\left(\sqrt{1-\left(1-\mu^{2}\right) \frac{r^{2}}{a^{2}}}\right) H\left(\mu-\sqrt{1-\frac{a^{2}}{r^{2}}}\right) .
$$

For the case that $\psi_{a}(\mu)=1 /(4 \pi)$, the scalar flux is

$$
\phi(r) \equiv 2 \pi \int_{-1}^{1} \psi(r, \mu) d \mu=\frac{1}{2}\left(1-\sqrt{1-\frac{a^{2}}{r^{2}}}\right), a \leq r \leq b .
$$

5. Representations in other coordinate systems. In order to use (2.15), (3.10), and (4.3) as tests for the ray effect errors in the scalar flux which is calculated in the cartesian or the cylindrical coordinate systems, these equations need to be expressed in cartesian and cylindrical coordinates. The desired equations for the scalar flux can be derived by the substitution of $r=\sqrt{x^{2}+y^{2}+z^{2}}$ for cartesian coordinates, and by the substitution of $r=\sqrt{\rho^{2}+z^{2}}$ for cylindrical coordinates into these equations.

The formulae (2.10), (2.14), (3.7), (3.8), (3.9), and (4.2) can also be used to test angular fluxes which are calculated in the cartesian or the cylindrical coordinate systems by expressing them in the coordinates of those systems. The relationship between the spherical angle $\mu$ and the cartesian angles $\left(\Omega_{x}, \Omega_{y}, \Omega_{z}\right)$ is $\mu=$ $\left(x \Omega_{x}+y \Omega_{y}+z \Omega_{z}\right) / \sqrt{x^{2}+y^{2}+z^{2}}$. Similarly, the spherical angle $\mu$ and the cylindrical angles $\left(\cos (\omega), \Omega_{z}\right)$ are related by $\mu=\left(\rho \sqrt{1-\Omega_{z}^{2}} \cos (\omega)+z \Omega_{z}\right) / \sqrt{\rho^{2}+z^{2}}$, where $\cos (\omega)$ is the cosine of the angle between $\vec{\rho}$ and the projection of $\Omega$ on the $x y$ plane [1].

6. Acknowledgments. I would like to thank M. Prasad for showing me Problem $\mathbf{C}$ and for sharing with me his work on characteristic methods.

\section{REFERENCES}

[1] E. E. LEWIS AND W. F. MILLER, Computational Methods of Neutron Transport, John Wiley, New York, 1984.

[2] M. Prasad, Laser Program Annual Report, UCRL 50021-86, p. 2-101. 